\title{
Characterizations of a CERN NbTi Reference Wire at LBNL*
}

\author{
A. Godeke ${ }^{\dagger}$ D. R. Dietderich, H. C. Higley, and N. L. Liggins \\ Ernest Orlando Lawrence Berkeley National Laboratory, One Cyclotron Rd, Berkeley, CA 94720, USA
}

February 1, 2007

\begin{abstract}
We report on the measurement of two NbTi reference samples, obtained from CERN through BNL, wire number 02R00056A01UX.265. The purpose of these characterizations is two-fold: 1) To establish an inter-laboratory comparison of short sample tests - and analysis - between LBNL, BNL and Fermilab within the LARP collaboration. Due to differences in reaction and mounting procedures and their resulting strain differences, $\mathrm{Nb}_{3} \mathrm{Sn}$ is not suited for this purpose. 2) To determine the accuracy of LBNL's system with regard to magnetic field, temperature and current after a major system overhaul. The results show that at $\mu_{0} H=8 \mathrm{~T}$, the critical current result falls within the standard deviation of the CERN database, whereas at $\mu_{0} H=5 \mathrm{~T}$, it falls outside the CERN standard deviation, but within the range of critical current values that are reported by CERN for this wire. It is concluded that the LBNL results fall within the characterization variance as reported in the CERN database.
\end{abstract}

\section{System changes}

LBNL's short sample test facility has been substantially modified. Improvements involved the commissioning of a new system control and data acquisition system, a new magnet power supply, a new nano-volt meter and improved wiring. The data acquisition software was developed by Dr. B. ten Haken at the University of Twente, the Netherlands, and has gone through more than 15 years of development. Efforts were made to improve system performance with respect to accuracy, noise, ease of operation, consistent data storage and measurement. Voltage current characterizations and data reduction are now almost fully automated, yielding consistency, ease of operation and high sample throughput.

\section{Voltage-current results}

Measurements of electric field as a function of current are best represented on a double logarithmic scale since physics, through the Anderson-Kim flux creep model [1,2], dictates linearity on a double logarithmic scale [3]:

$$
E=E_{c}\left(\frac{J}{J_{c}(H, T)}\right)^{n(H, T)} .
$$

Measurement errors are effectively amplified on a double logarithmic plot through non-linearities that can arise from offset drift, sample heating, and current re-distribution and entrance effects (i.e. current sharing with the matrix in general). One can argue whether some non-linearities should be seen as a property of a wire, as opposed to probing solely the properties of the superconducting volume inside the wire. Usually, however, non-linearities in a double logarithmic plot can be traced back to a faulty measurement [3].

Two NbTi samples were characterized. Sample $a$ was used to analyze and test the system, and optimize performance with respect to noise. Measurements are performed in DC mode, to prevent inductive voltage pickup and AC artifacts, creating a better defined characterization. The measured electric field as a function of sample current for both samples is depicted on a double logarithmic scale in Figure 1. Two voltage tap pairs were used over a 5 turn and a 7 turn section (i.e. about $0.5 \mathrm{~m}$ and $0.7 \mathrm{~m}$ sample length respectively). The voltages from both pairs are plotted in

\footnotetext{
*Supported by the Director, Office of Science, High Energy Physics, U.S. Department of Energy under contract No. DE-AC02-05CH11231

${ }^{\dagger}$ Electronic address: agodeke@lbl.gov
} 


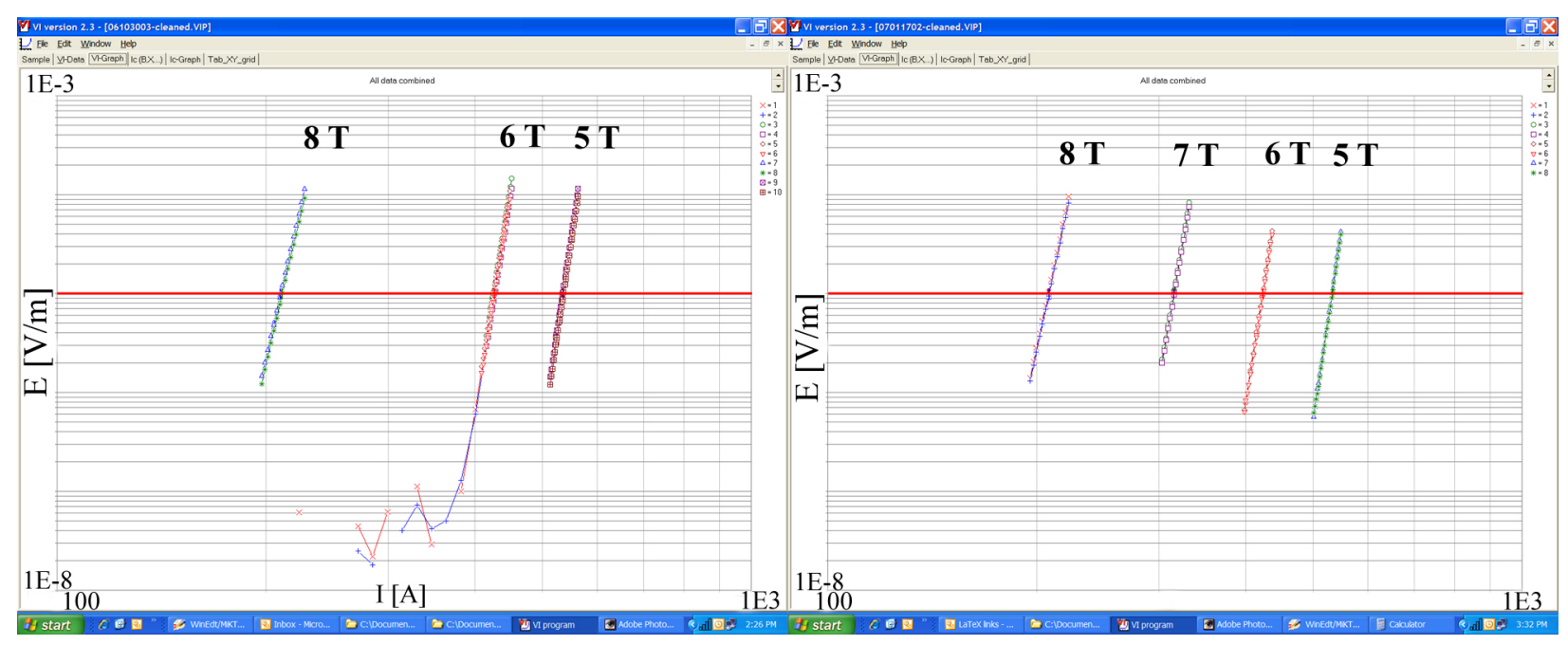

Figure 1: Electric field as function of current for sample $a$ (left) and sample $b$ (right).

Table 1: Critical current results

\begin{tabular}{|c|c|c|c|c|c|c|c|c|c|}
\hline \multirow[b]{3}{*}{$\mu_{0} H$} & \multirow{2}{*}{\multicolumn{2}{|c|}{$\begin{array}{c}\text { CERN } \\
T=4.222 \mathrm{~K}\end{array}$}} & \multirow{2}{*}{\multicolumn{3}{|c|}{$\begin{array}{c}\text { Sample } a: 5 \text { turn } / 7 \text { turn } \\
T=\text { not recorded }\end{array}$}} & \multicolumn{4}{|c|}{ Sample $b$ : 5 turn $/ 7$ turn } \\
\hline & & & & & & \multicolumn{3}{|c|}{$T=4.233 \mathrm{~K}$} & $4.222 \mathrm{~K}$ \\
\hline & $\begin{array}{c}I_{c} \\
10^{-14} \Omega m\end{array}$ & $n$ & $\begin{array}{c}I_{c} \\
10^{-5} \mathrm{~V} / \mathrm{m}\end{array}$ & $n$ & $\begin{array}{c}I_{c} \\
10^{-14} \Omega \mathrm{m}\end{array}$ & $\begin{array}{c}I_{c} \\
10^{-5} \mathrm{~V} / \mathrm{m}\end{array}$ & $n$ & $\begin{array}{c}I_{c} \\
10^{-14} \Omega \mathrm{m}\end{array}$ & $\begin{array}{c}I_{c} \\
10^{-14} \Omega m\end{array}$ \\
\hline$[\mathrm{T}]$ & [A] & & [A] & & [A] & {$[\mathrm{A}]$} & & {$[\mathrm{A}]$} & {$[\mathrm{A}]$} \\
\hline 5 & 538.8 & 52 & $535 / 537$ & $47 / 47$ & $535 / 537$ & $532 / 533$ & $49 / 48$ & $532 / 533$ & $534 / 535$ \\
\hline 6 & 426.2 & 48 & $425 / 427$ & $44 / 44$ & $423 / 424$ & $423 / 423$ & $46 / 45$ & $421 / 421$ & $423 / 423$ \\
\hline 7 & 313.6 & 43 & & & & $315 / 315$ & $41 / 41$ & $311 / 311$ & $312 / 313$ \\
\hline 8 & 201.7 & 35 & $210 / 211$ & $30 / 30$ & $203 / 204$ & $208 / 208$ & $33 / 33$ & $202 / 202$ & $203 / 204$ \\
\hline
\end{tabular}

Figure 1. LBNL's data acquisition software uses a selectable electric field criterion since this is less ambiguous than a resistive criterion since the latter involves a choice with respect to the area to be used (e.g. total or non-Cu). A usual criterion for measurements on a barrel is $10^{-5} \mathrm{~V} / \mathrm{m}$. Data are collected over one electric field decade above and below this criterion, thereby preventing the collection of irrelevant data far below the transition and thus saving time. This range was extended down for the transition at $\mu_{0} H=6 \mathrm{~T}$ for sample $a$, demonstrating a noise level of $E \cong 10^{-7} \mathrm{~V} / \mathrm{m}$, or about $50 \mathrm{nV}$.

\section{Critical current results}

Critical current is determined by fitting the measured electric field as function of current data to relation (1) (replacing $J$ by $I$ ) over a selectable electric field range and calculating the intersect with the electric field criterion. Here, $E(I)$ data that fall within a window $E_{c} / 3<E_{c}<3 E_{c}$ were fitted to (1) to calculate $I_{c}$ at $E_{c}=10^{-5} \mathrm{~V} / \mathrm{m}$. The resulting critical currents are summarized in Table 1, together with the reference results from CERN [4]. The $E(I)$ data are linear on a double logarithmic plot (Figure 1) and thus follow (1). The critical current results, derived using an electric field criterion, can thus be translated to a resistive criterion through a combination of (1) with Ohm's law, yielding:

$$
I_{\rho}=\left(\frac{\rho I_{c}^{n}}{E_{c} A}\right)^{\frac{1}{n-1}},
$$




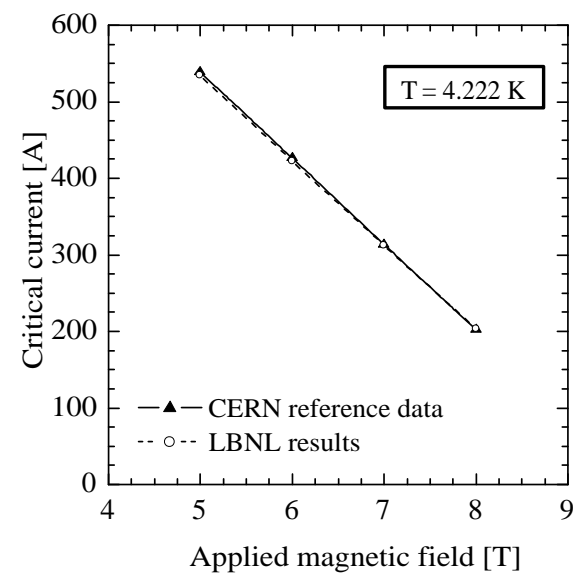

Figure 2: Critical current as a function of magnetic field at $T=4.222 \mathrm{~K}$ for the CERN reference data and LBNL results for the 5 turn section of sample $b$.

where $I_{\rho}$ is the critical current at a resistive criterion $\rho$. These calculated critical currents at a criterion $\rho=10^{-14} \Omega \mathrm{m}$, using a wire diameter of $0.825 \mathrm{~mm}$, are also given in Table 1.

\section{Temperature corrections}

The Helium bath temperature during measurement of sample $a$ was not recorded. LBNL's system is equipped with vapor cooled current leads. The Helium gas flow through these leads is typically adjusted to run at an overpressure of 0.15 to 0.2 psi. For now, the bath temperature is influenced by variations in atmospheric pressure. Improvements are planned to register the Helium bath temperature during all characterization runs.

For sample $b$, the bath temperature was recorded using two calibrated Carbon Glass Resistor (CGR) thermometers positioned on the top $\left(T_{t o p}\right)$ and bottom $\left(T_{b o t}\right)$ flange of the magnet. A readout at the start of the measuring session yielded $T_{\text {top }}=4.231 \mathrm{~K}$ and $T_{\text {bot }}=4.236 \mathrm{~K}$. The sample temperature is taken as the average between these two measurements.

The CERN reference data are given at a temperature $T=4.222 \mathrm{~K}$ and the data for sample $b$ are corrected for this small discrepancy in temperature. Ideally, the samples should be parameterized using well established scaling relations [5] but the required scaling parameters cannot be accurately determined due to the limited data range. Also, since the temperature error is small, a limited range - linear - correction will be sufficient [6]:

$$
I_{c}(4.222 \mathrm{~K})=I_{c}\left(T_{b a t h}\right)\left(\frac{T_{c}(H)-4.222}{T_{c}(H)-T_{b a t h}}\right),
$$

where:

$$
T_{c}(H)=9.2\left(1-\frac{\mu_{0} H}{14.5}\right)^{0.59}
$$

Note that relation 4 implicitly assumes $T_{c}(0)=9.2 \mathrm{~K}, \mu_{0} H_{c 2}(0)=14.5 \mathrm{~T}$ and $H_{c 2}(T) / H_{c 2}(0)=1-\left(T / T_{c}(0)\right)^{1.7}$. These re-normalized data are also given in Table 1 and the resulting $I_{c}(H)$ data for the 5 turn section are, together with the CERN reference data, plotted in Figure 2. Corrections for self-field were considered, but these will always be subjective and only become relevant below $\mu_{0} H \lesssim 3$ T. No self-field corrections were therefore applied on the results.

\section{Discussion}

When comparing the CERN reference data with LBNL's measurements (Table 1, Figure 2), it is seen that the agreement is good, but not perfect. The CERN reference data has a standard deviation of $1.7 \mathrm{~A}$ at $8 \mathrm{~T}$ and $2.5 \mathrm{~A}$ at $5 \mathrm{~T}$ [4]. 
LBNL's results therefore fall within the standard deviation at $8 \mathrm{~T}$, but just below the standard deviation at $5 \mathrm{~T}$. LBNL's measurement at $5 \mathrm{~T}$ is, however, situated significantly above the lowest value reported in the CERN statistics, which is reported as $531.9 \mathrm{~A}$.

It is interesting to note that LBNL's results show a slightly different slope in the $I_{c}(H)$ graph (Figure 2). Both magnetic field errors and current errors will cause a proportional shift in the $I_{c}(H)$ results and can thus be ruled out. From a measurement point of view, a slope change can arise either from a temperature error, or from a difference in critical current criterion (i.e. resistive versus an electric field criterion). From a physical point of view, such a slope change can arise from a difference in the effective pinning force and/or effective upper critical magnetic field. Since both LBNL's thermometers agree within $5 \mathrm{mK}$ (which can be attributed to the Helium column above the bottom thermometer) and critical current criteria are used consistently, it is concluded that the most probably cause for the slope difference is slight differences in wire physical properties.

\section{References}

[1] P. W. Anderson, Phys. Rev. Lett. 9, 309 (1962).

[2] Y. B. Kim, C. F. Hempstead, and A. R. Strnad, Phys. Rev. Lett. 9, 306 (1962).

[3] A. Godeke, Performance Boundaries in $\mathrm{Nb}_{3} \mathrm{Sn}$ Superconductors, PhD thesis, Univ. of Twente, Enschede, The Netherlands, 2005.

[4] T. Boutboul, Technical report, CERN, 2006.

[5] L. Bottura, IEEE Trans. Appl. Supercond. 10, 1054 (2000).

[6] A. Ghosh, Technical report, BNL, 2006. 